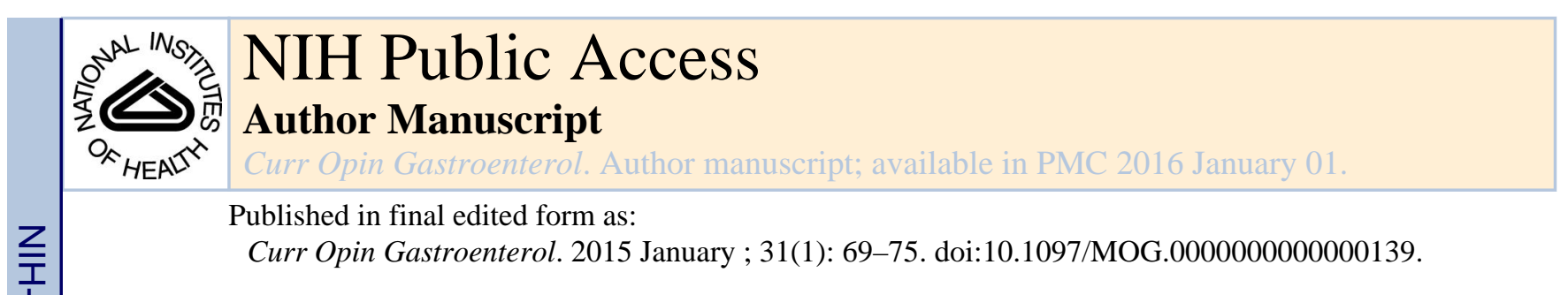

\title{
The gut microbiome in health and in disease
}

\author{
Andrew B. Shreiner ${ }^{1}$, John Y. Kao ${ }^{1}$, and Vincent B. Young ${ }^{2}$ \\ ${ }^{1}$ Division of Gastroenterology, Department of Internal Medicine, University of Michigan Medical \\ School \\ ${ }^{2}$ Division of Infectious Diseases, Department of Internal Medicine, University of Michigan Medical \\ School
}

\begin{abstract}
Purpose of review-Recent technological advancements and expanded efforts have led to a tremendous growth in the collective knowledge of the human microbiome. This review will highlight some of the important recent findings in this area of research.
\end{abstract}

Recent findings-Studies have described the structure and functional capacity of the bacterial microbiome in the healthy state and in a variety of disease states. Downstream analyses of the functional interactions between the host and its microbiome are starting to provide mechanistic insights into these interactions. These data are anticipated to lead to new opportunities for diagnosis, prognosis, and treatment of a variety of human diseases.

Summary-There is a fast growing collection of data describing the structure and functional capacity of the microbiome in a variety of conditions available to the research community for consideration and further exploration. Ongoing efforts to further characterize the functions of the microbiome and the mechanisms underlying host-microbe interactions will provide a better understanding of the role of the microbiome in health and disease.

\section{Keywords}

Gut microbiome; microbiota; dysbiosis; health; disease

\section{INTRODUCTION}

The human microbiome is composed of bacteria, archaea, viruses and eukaryotic microbes that reside in and on our bodies. These microbes have tremendous potential to impact our physiology, both in health and in disease. They contribute metabolic functions, protect against pathogens, educate the immune system, and, through these basic functions, affect directly or indirectly most of our physiologic functions.

The study of the human microbiome has been furthered by technological advancements for performing culture-independent analyses (1). In most studies, the bacterial constituents of a

Corresponding author info: Vincent B. Young, M.D., Ph.D., Division of Infectious Diseases, Department of Internal Medicine, Department of Microbiology \& Immunology, University of Michigan Health System, 1520B MSRB I SPC 5666, 1150 W. Medical Center Dr., Ann Arbor, MI 48109-5666, USA. Tel: 734-764-2237; Fax: 734-763-4168; youngvi@umich.edu. 
microbial population are identified by sequencing of the $16 \mathrm{~S}$ rRNA-encoding gene (hereafter, 16S) followed by comparison to known bacterial sequence databases. Metagenomic analysis by sequencing all microbial DNA in a complex community has the additional advantage of assessing the genetic potential of the microbial population. Other methodologies to analyze the microbial transcriptome, proteome, and metabolome provide additional information at successive levels of microbial physiology (2). We will not go into further detail on specific technical considerations in this space, but interested readers are referred to recent review articles (3-5).

Great progress in characterizing the structure of the microbiome recently has paved the way for ongoing and future studies on the functional interactions between the microbiota and the host. Studies on the function of the microbiota will be critical to understanding the role of the microbiota in human homeostasis and disease pathogenesis. In this review, we will discuss recent advancements in our understanding of the structure and function of the microbiome associated with the healthy state and with specific diseased states.

\section{Accumulation of data on the human microbiome}

The tremendous expansion of information collected on the human microbiome in recent years is highlighted by data generated through several large-scale endeavors to characterize the human microbiome, namely the European Metagenomics of the Human Intestinal Tract (MetaHIT) and the NIH-funded Human Microbiome Project (HMP) (6, 7). In 2010, the initial MetaHIT consortium study reported sequencing 3.3 million non-redundant fecal microbial genes, representing almost 200 times the quantity of microbial DNA sequences reported in all previous studies (7). In July 2014, a combined set of metagonomic sequencing data from 1267 gut metagenomes from 1070 individuals, including 760 European samples from MetaHIT, 139 American samples from HMP and 368 Chinese samples from a large diabetes study, was published with a non-redundant gene catalog of 9.8 million microbial genes $(* 8)$. Each sample contained about 750,000 genes or about 30 times the number of genes in the human genome, and less than 300,000 genes were shared by greater than $50 \%$ of individuals. The majority of the new genes identified in this latest study were relatively rare, found in less than $1 \%$ of individuals. This collection is thought to contain nearly a complete set of genes for most human gut bacteria and illustrates the quantity and variability of the human microbiome.

\section{Structure and dynamics of the healthy adult microbiota}

Characterization of the microbiome in healthy individuals is an important initial step in understanding the role of the microbiome in contributing to health and disease. Healthy adult humans each typically harbor more than 1000 species of bacteria belonging to a relatively few known bacterial phyla with Bacteroidetes and Firmicutes being the dominant phyla (9). The microbiota of the gut is quite diverse (Table 1) compared to other body sites, and there is considerable variation in the constituents of the gut microbiota among apparently healthy individuals (10). As a way of accounting for the microbial variability among healthy individuals, researchers have tried to identify certain stable patterns of microbial populations in the human population (11). Data from the HMP was used to identify community types at different body sites based on statistical analysis of the 
configuration of multiple bacterial taxa $(* 12)$. Four distinct communities were found in the stool, and metadata factors associated with community types included breastfeeding, gender and education. Interestingly, community types in the oral cavity were predictive of those in the stool though the specific constituents were different. In a recent study of 37 healthy American adults not taking antibiotics, greater than $70 \%$ of fecal bacterial species within an individual were stable over 1 year, and few additional changes were measured out to 5 years $(* 13)$. Calculations showed that species were likely stable over decades if not for an individual's entire lifetime as evidenced by species shared with adult family members but not with unrelated individuals. While we have gained a deep understanding of the structure and dynamics of the microbiome in healthy humans, this effort is complicated by significant variability in the population, modest variability over time within an individual, and uncertainty over the most meaningful ways to characterize the microbiota.

\section{Metabolic functions of the microbiota}

After characterizing microbial community membership and dynamics, it is critical to understand the functional activities that ultimately affect host physiology. Gut microbiota are integral to host digestion and nutrition, and they can generate nutrients from substrates that are otherwise indigestible by the host. For instance, xyloglucans are commonly found in dietary vegetables such as lettuce and onions, and the capacity for microbial digestion of xyloglucans was recently mapped to a single locus in a certain species of Bacteroides (*14). The ability to digest xyloglucans was shown to be a relatively rare trait in members of the phylum Bacteroidetes, and the importance of this capability to the human host was demonstrated by analysis of a public metagenome database showing that $92 \%$ of individuals contained at least one of these rare Bacteroides species capable of digesting xyloglucans. These findings illustrate how humans have cultivated mutually beneficial relationships with gut microbiota with implications for diet and nutrition.

Microbes liberate short chain fatty acids (SCFA) from indigestible dietary fibers, and SCFA are an important energy source for intestinal mucosa and critical for modulating immune responses and tumorigenesis in the gut. The role of butyrate, an abundant bioactive SCFA in the gut, plays a complex role in colon cancer that seems to be concentration and context dependent as illustrated by two recent preclinical studies. Butyrate was reported to promote tumorigenesis in transgenic mice with combined tumor suppressor gene (APC) mutation and mismatch repair gene (MSH2) deficiency, because tumor formation was decreased by antibiotic treatment or low carbohydrate diet, both of which decrease butyrate levels, and increased by feeding butyrate to antibiotic-treated mice $(* 15)$. Conversely, butyrate was reported to inhibit tumorigenesis, because mice deficient in Grp109a, a receptor for butyrate, had increased tumorigenesis promoted by inflammatory stimuli or APC mutation and signaling through Grp109a inhibited tumorigenesis induced by these stimuli (*16). Further investigations into the role of butyrate produced by microbiota in colitis and colorectal cancer are awaited. The studies discussed in this section demonstrate the need to assess the function of the microbiota in order to better understand its role in health and disease. 


\section{Host-microbe interactions on the immune system}

Interactions between the microbiota and the host immune system are numerous, complex, and bidirectional. The immune system must learn to tolerate the commensal microbiota and respond appropriately to pathogens, and in turn the microbiota is integral to educating the immune system to function properly. Here we highlight studies that describe how members of the microbial community promote the differentiation of anti-inflammatory regulatory $\mathrm{T}$ cells (Treg) to illustrate how the microbiota can influence immune homeostasis. A series of experiments showed that collections of nonpathogenic species of Clostridia from clusters IV, XIVa and XVIII, isolated after application of a series of non-specific selection steps, were capable of inducing colonic Treg, and one mechanism may involve the production of butyrate that affects epigenetic control of the Foxp3 promoter that controls Treg development $(17, * * 18,19-22)$. In germ-free mice that do not contain endogenous microbiota, another group also devised a novel method to screen human fecal samples for bacterial strains able to promote Treg development, and they noted this functional capacity in more strains than anticipated $(* 23)$. While not discussed here, there is evidence detailing host-microbe interactions that influence immune functions at all levels from the initial innate defenses to the complex acquired responses discussed in this section (24). There is great interest in elucidating how the microbiota can influence immune homeostasis inside and outside the gut, as this process has important implications for the pathogenesis and treatment of inflammatory disorders and a growing list of diseases linked to inflammation.

\section{The role of the microbiota in specific diseases and conditions}

The above sections have described some of the many ways that the microbiota can influence human physiology, and it is no surprise that there is great interest in studying microbiota changes associated with diseased states, often referred to as dysbiosis (Table 1). However, the relationship between dysbiosis and disease pathogenesis is uncertain in the majority of examples at this time. It is often not clear what microbiota changes associated with disease are meaningful and distinguishing between cause and effect is inherently challenging. While it is intriguing to speculate that dysbiosis may cause disease as we learn more about how the microbiota can influence the host, it is also noted that the diseased state can lead to changes to the microbiota through various mechanisms, including changes in eating habits and bowel function as well as through the addition of medications such as antibiotics. In this section, we highlight a few of the recent findings on the role of the microbiota in particular diseases or conditions, but we cannot touch on all of the emerging findings in a multitude of other diseases both inside and outside the gut, including but not limited to rheumatoid arthritis (25), colorectal cancer (26), obesity (27), and diabetes (28).

\section{Cardiovascular disease}

There is growing interest in a link between microbiota and cardiovascular disease based on data showing microbial metabolism of dietary phosphatidylcholine into the proatherosclerotic metabolite trimethylamine- $N$-oxide (TMAO) (29). A recent study of healthy patients challenged with dietary phosphatidylcholine showed increased plasma levels of TMAO that were suppressed by prior treatment with antibiotics. They also found that plasma TMAO levels were associated with increased risk for cardiovascular events in 
patients with cardiovascular disease risk factors $(* 30)$. In another study, the same group showed that healthy human volunteers who maintained a vegan diet, as opposed to those on omnivorous diets, did not demonstrate increased plasma TMAO levels after dietary phosphatidylcholine challenge, and this trait was associated with specific fecal microbiota compositional states $(* 31)$. Thus, there is much interest in this microbiota-dependent pathway that may offer diagnostic and therapeutic potential for cardiovascular disease.

\section{Irritable bowel disease and the microbiota-gut-brain axis}

A role for the microbiota in irritable bowel syndrome (IBS) is suspected, though unproven, and therapies that alter the microbiota, including dietary changes, probiotics, and antibiotics, have shown encouraging, though inconsistent, results (32). In two reports describing the results of dietary intervention with a low FODMAP (fermentable oligosaccharides, disaccharides, monosaccharides and polyols) diet consisting of restricted intake of certain fermentable substrates compared to a typical Australian diet in a small number of Australian patients with IBS, the low FODMAP diet improved symptoms and resulted in changes in the gut microbiota, including reductions in putatively healthy bacteria, such as those in butyrateproducing Clostridium cluster XIVa (33, *34). One proposed pathway involved in IBS is through a microbiota-gut-brain axis, linking changes in the gut to symptom perception in the central nervous system. An interesting recent report demonstrated that the intake of a probiotic-rich fermented milk product resulted in alterations in brain activity in response to visual emotional stimuli as measured by functional magnetic resonance imaging as compared to the intake of a control product (35). The study of IBS is challenging due to the lack of specific diagnostic tests and the possibility of heterogeneous etiologies. There may be a subset of patients where microbiota changes are particularly important and for whom therapies to affect microbiota composition and function may be beneficial.

\section{Clostridium difficile infection}

Clostridium difficile infection (CDI) is the prime example of a human disease that develops as a result of critical changes to the gut microbiota and is effectively treated by microbiotabased therapy (36). A meta-analysis to review the use of fecal microbiota transplant (FMT) for prevention of recurrent CDI identified 11 studies with 273 patients through 2012; the overall resulting efficacy was about $90 \%$ and no substantial FMT-related adverse events were reported (37). In a prospective clinical trial, patients with recurrent CDI were randomly assigned to one of three treatment groups, standard vancomycin therapy, vancomycin therapy followed by bowel lavage, and vancomycin therapy followed by bowel lavage and subsequent infusion of donor stool into the duodenum (**38). The study was stopped early after interim analysis due to the superiority of FMT. Analysis of the fecal microbiota of patients who were treated with FMT for recurrent CDI demonstrated that the post-transplant microbiota of the recipients becomes more similar to that of the donor. After FMT the recipient microbiota was characterized by increased diversity, increased abundance of various Firmicutes and Bacteroidetes, and decreased abundance of Proteobacteria $(* 39)$. In a proof-of-principle study, two patients with CDI refractory to antibiotics were successfully treated with a stool substitute consisting of 33 strains of bacteria isolated and cultured from a healthy donor $(* 40)$. In this study, the stool substitute was delivered with colonoscopy, but it does provide promise for using a selected population of bacteria that could be prepared in a 
laboratory. Collectively, these studies strongly support the treatment of recurrent CDI with microbiota-based therapies.

While susceptibility to CDI after antibiotic use is associated with decreased microbiota diversity, little is known about the functional difference in the microenvironment that permits CDI. In preclinical studies, the microbiota changes that occurred after antibiotic treatment in mice susceptible to $C$. difficile were accompanied by changes in the metabolome that supported $C$. difficile germination and growth $(* 41)$. In the antibiotictreated mice, primary bile acids that support $C$. difficile germination and certain carbohydrates that support $C$. difficile growth were present at significantly increased levels compared to control C. difficile-resistant mice. This study, and others, are helping to unravel the mechanism by which antibiotic-induced changes to the microbiota contribute to CDI, potentially leading to novel therapies for this burdensome disease.

\section{Inflammatory bowel disease}

Inflammatory bowel diseases (IBD) are characterized by inappropriate inflammation in the gut resulting from a combination of environmental and genetic risk factors. Targets of the inflammatory response include the commensal microbiota, and IBD are associated with alterations in the gut microbiota, though it is not clear if microbial changes contribute to disease pathogenesis or develop as a result of local inflammation (42). Here, we highlight two recent publications describing the microbiome and host response measured in treatment naïve pediatric patients with newly diagnosed IBD that provide early findings not confounded by anti-inflammatory therapies.

In the first report, 447 treatment naïve pediatric Crohn's disease (CD) patients and 221 controls were included in a study where the microbiome from multiple sites was characterized by $16 \mathrm{~S}$ sequence analysis $(* * 43)$. Multivariate analysis identified microbial taxa significantly associated with disease phenotype in ileal and rectal samples but not from stool samples. The microbiome of CD patients had a lower diversity, increased abundances of Enterobacteriaceae, Pasteurellaceae, Fusobacteriaceae, Neisseriaceae, Veillonellaceae, and Gemellaceae, and decreased abundances of Bifidobacteriaceae, Erysipelotrichaceae, Clostridiales, and Bacteroidales. Using these microbiome-disease associations, they formulated a microbial dysbiosis index that showed a strong positive correlation with clinical disease activity (PDCAI) and a negative correlation with species richness (Table 1). In addition, microbiome comparison between $\mathrm{CD}$ patients with and without antibiotic exposure revealed that antibiotic use amplifies the microbial dysbiosis associated with $\mathrm{CD}$. Of note, the authors demonstrate that many of their observations were only seen when analyzing hundreds of samples, emphasizing the importance of large-scale studies.

In the next report, host gene expression and the microbiome were characterized from the ileum of treatment naïve pediatric patients with ileal CD (iCD), colonic $\mathrm{CD}(\mathrm{cCD})$, and ulcerative colitis (UC) as well as controls (**44). A core iCD gene expression signature of 1281 genes was identified by comparing iCD and control groups. Upregulated genes included those induced by bacterial products and proinflammatory signals, and downregulated genes included nuclear receptors involved in metabolic pathways and antiinflammatory signaling. Interestingly, the cCD group, including those without any 
microscopic inflammation, had a similar expression pattern in the ileum to the iCD group, and this pattern was different from control and UC groups, indicating a core CD gene expression profile in the ileum independent of inflammation. Similarly, iCD and cCD groups had similar profiles of dysbiosis that was different from control and UC groups. Of note, an increase of antimicrobial dual oxidase (DUOX2) expression was detected in association with an expansion of Proteobacteria in both UC and CD, while expression of lipoprotein APOA1 gene was downregulated and associated with CD-specific alterations in Firmicutes. Finally, multivariate analysis showed correlations between bacterial taxa, gene expression and clinical disease activity scores in CD patients irrespective of the presence of ileal inflammation, and a prediction model based on gene expression, microbe abundance and clinical factors outperformed clinical disease activity scores alone in predicting response to therapy.

These studies provide additional information towards understanding a potential role of the microbiota in the pathogenesis of IBD that has not been established to date. This stands in contrast to CDI where microbiota alterations clearly increase the risk for disease and restoration of a diverse microbiota with FMT clearly prevents disease recurrence in most patients. Early efforts to utilize FMT for the treatment of IBD have been rather disappointing $(45,46)$. It seems quite likely that the role of microbiota in IBD, and other multigenic traits, will prove to be more complex and depend on specific genetic susceptibilities and certain environmental factors. Therefore, it will be important to further characterize the microbiota population and its functions at various time points in IBD development in conjunction with assessments of host susceptibility, host response and environmental exposures.

\section{CONCLUSION}

This is a very exciting time in the study of the microbiome due to recent technological advancements and rapidly accumulating knowledge. Large-scale efforts have described in great detail the constituents of the healthy human microbiome and its functional capacity, and these studies are followed by similar characterizations of the microbiome in particular diseased states. The critical next stage that is underway is to investigate further the functions of the microbiome. These studies will provide further insight into the host-microbiome interactions that contribute to health and disease, and eventually lead to therapies targeting the microbiome to maintain health and to treat a variety of diseases.

\section{ACKNOWLEDGEMENTS}

- None

Disclosure of funding: This work was funded by the NIH: DK094775 (ABS), DK087708 (JYK), DK083993 (VBY) and AI090871 (VBY).

\section{REFERENCE SECTION}

1. Robinson CJ, Bohannan BJ, Young VB. From structure to function: the ecology of host-associated microbial communities. Microbiol Mol Biol Rev. Sep; 2010 74(3):453-76. PubMed PMID: 20805407. Pubmed Central PMCID: 2937523. Epub 2010/09/02. eng. [PubMed: 20805407] 
2. Bassis, CM.; Young, VB.; Schmidt, TM. Methods for Characterizing Microbial Communities Associated With the Human Body. In: Fredricks, DN., editor. The Human Microbiota: How Microbial Communities Affect Health and Disease. John Wiley \& Sons, Inc.; Hoboken, New Jersey: 2013. p. 51-74.

3. Di Bella JM, Bao Y, Gloor GB, et al. High throughput sequencing methods and analysis for microbiome research. Journal of microbiological methods. Dec; 2013 95(3):401-14. PubMed PMID: 24029734. [PubMed: 24029734]

4. Kumar R, Eipers P, Little RB, et al. Getting started with microbiome analysis: sample acquisition to bioinformatics. Current protocols in human genetics / editorial board. 2014; 82:18. Jonathan L Haines [et al]. 8 1- 8 29. PubMed PMID: 25042718.

5. Morgan XC, Huttenhower C. Meta'omic analytic techniques for studying the intestinal microbiome. Gastroenterology. May; 2014 146(6):1437-48. e1. PubMed PMID: 24486053. [PubMed: 24486053]

6. Human Microbiome Project C. A framework for human microbiome research. Nature. Jun 14; 2012 486(7402):215-21. PubMed PMID: 22699610. Pubmed Central PMCID: 3377744. [PubMed: 22699610]

7. Qin J, Li R, Raes J, et al. A human gut microbial gene catalogue established by metagenomic sequencing. Nature. Mar 4; 2010 464(7285):59-65. PubMed PMID: 20203603. Pubmed Central PMCID: 3779803. [PubMed: 20203603]

*8. Li J, Jia H, Cai X, et al. An integrated catalog of reference genes in the human gut microbiome. Nature biotechnology. Jul 6.2014 PubMed PMID: 24997786. This article reports the most current and largest compilation to date of bacterial sequences combined from several large-scale metagenomic studies on the human gut microbiome. This catalog is an important resource for further exploration.

9. Lozupone CA, Stombaugh JI, Gordon JI, et al. Diversity, stability and resilience of the human gut microbiota. Nature. Sep 13; 2012 489(7415):220-30. PubMed PMID: 22972295. Pubmed Central PMCID: 3577372. [PubMed: 22972295]

10. Human Microbiome Project C. Structure, function and diversity of the healthy human microbiome. Nature. Jun 14; 2012 486(7402):207-14. PubMed PMID: 22699609. Pubmed Central PMCID: 3564958. [PubMed: 22699609]

11. Arumugam M, Raes J, Pelletier E, et al. Enterotypes of the human gut microbiome. Nature. May 12; 2011 473(7346):174-80. PubMed PMID: 21508958. Pubmed Central PMCID: 3728647. [PubMed: 21508958]

*12. Ding T, Schloss PD. Dynamics and associations of microbial community types across the human body. Nature. May 15; 2014 509(7500):357-60. PubMed PMID: 24739969. The authors apply bioinformatics techniques to publicly available data sets demonstrating that shared microbial community types can be identified in spite of considerable variation between subjects. If different community types have biologic significance, there are implications for assessing and treating patients. [PubMed: 24739969]

*13. Faith JJ, Guruge JL, Charbonneau M, et al. The long-term stability of the human gut microbiota. Science. Jul 5.2013 341(6141):1237439. PubMed PMID: 23828941. Pubmed Central PMCID: 3791589. This study characterizes the human gut microbiota over time, and it provides important new evidence that the microbiota of an individual is quite stable over years and likely decades. [PubMed: 23828941]

*14. Larsbrink J, Rogers TE, Hemsworth GR, et al. A discrete genetic locus confers xyloglucan metabolism in select human gut Bacteroidetes. Nature. Feb 27; 2014 506(7489):498-502. PubMed PMID: 24463512. This study demonstrates that a common dietary fiber is metabolized by a select few bacterial species that are commonly present in the human gut microbiota, illustrating the concept of human-microbe symbiosis. [PubMed: 24463512]

*15. Belcheva A, Irrazabal T, Robertson SJ, et al. Gut microbial metabolism drives transformation of msh2-deficient colon epithelial cells. Cell. Jul 17; 2014 158(2):288-99. PubMed PMID: 25036629. This study provides evidence that butyrate promotes colon tumorigenesis in a mouse model of APC gene mutation and MSH2 gene depletion. [PubMed: 25036629]

*16. Singh N, Gurav A, Sivaprakasam S, et al. Activation of Gpr109a, receptor for niacin and the commensal metabolite butyrate, suppresses colonic inflammation and carcinogenesis. Immunity. Jan 16; 2014 40(1):128-39. PubMed PMID: 24412617. This study provides evidence that 
butyrate inhibits colon tumorigenesis in mouse models of APC gene mutation or colitis. [PubMed: 24412617]

17. Arpaia N, Campbell C, Fan X, et al. Metabolites produced by commensal bacteria promote peripheral regulatory T-cell generation. Nature. Dec 19; 2013 504(7480):451-5. PubMed PMID: 24226773. Pubmed Central PMCID: 3869884. [PubMed: 24226773]

**18. Atarashi K, Tanoue T, Oshima K, et al. Treg induction by a rationally selected mixture of Clostridia strains from the human microbiota. Nature. Aug 8; 2013 500(7461):232-6. PubMed PMID: 23842501. Using a novel strategy to identify bacterial communities from the human gut microbiota that promote Treg development, the authors identify 17 strains of non-pathogenic Clostridia capable of inducing Treg. This approach is instructive for the rational design of probiotic therapies. [PubMed: 23842501]

19. Atarashi K, Tanoue T, Shima T, et al. Induction of colonic regulatory T cells by indigenous Clostridium species. Science. Jan 21; 2011 331(6015):337-41. PubMed PMID: 21205640. Pubmed Central PMCID: 3969237. [PubMed: 21205640]

20. Furusawa Y, Obata Y, Fukuda S, et al. Commensal microbe-derived butyrate induces the differentiation of colonic regulatory T cells. Nature. Dec 19; 2013 504(7480):446-50. PubMed PMID: 24226770. [PubMed: 24226770]

21. Narushima S, Sugiura Y, Oshima K, et al. Characterization of the 17 strains of regulatory T cellinducing human-derived Clostridia. Gut microbes. Mar 18.2014 5(3) PubMed PMID: 24642476.

22. Smith PM, Howitt MR, Panikov N, et al. The microbial metabolites, short-chain fatty acids, regulate colonic Treg cell homeostasis. Science. Aug 2; 2013 341(6145):569-73. PubMed PMID: 23828891. Pubmed Central PMCID: 3807819. [PubMed: 23828891]

23. Faith JJ, Ahern PP, Ridaura VK, et al. Identifying gut microbe-host phenotype relationships using combinatorial communities in gnotobiotic mice. Science translational medicine. Jan 22.2014 6(220):220ra11. PubMed PMID: 24452263. Pubmed Central PMCID: 3973144.These authors describe another unbiased novel method for identifying bacterial strains capable of inducing Treg.

24. Kamada N, Nunez G. Regulation of the immune system by the resident intestinal bacteria. Gastroenterology. May; 2014 146(6):1477-88. PubMed PMID: 24503128. Pubmed Central PMCID: 3995843. [PubMed: 24503128]

25. Scher JU, Sczesnak A, Longman RS, et al. Expansion of intestinal Prevotella copri correlates with enhanced susceptibility to arthritis. eLife. 2013; 2:e01202. PubMed PMID: 24192039. Pubmed Central PMCID: 3816614. [PubMed: 24192039]

26. Kostic AD, Chun E, Robertson L, et al. Fusobacterium nucleatum potentiates intestinal tumorigenesis and modulates the tumor-immune microenvironment. Cell host \& microbe. Aug 14; 2013 14(2):207-15. PubMed PMID: 23954159. Pubmed Central PMCID: 3772512. [PubMed: 23954159]

27. Le Chatelier E, Nielsen T, Qin J, et al. Richness of human gut microbiome correlates with metabolic markers. Nature. Aug 29; 2013 500(7464):541-6. PubMed PMID: 23985870. [PubMed: 23985870]

28. Karlsson FH, Tremaroli V, Nookaew I, et al. Gut metagenome in European women with normal, impaired and diabetic glucose control. Nature. Jun 6; 2013 498(7452):99-103. PubMed PMID: 23719380. [PubMed: 23719380]

29. Wang Z, Klipfell E, Bennett BJ, et al. Gut flora metabolism of phosphatidylcholine promotes cardiovascular disease. Nature. Apr 7; 2011 472(7341):57-63. PubMed PMID: 21475195. Pubmed Central PMCID: 3086762. [PubMed: 21475195]

*30. Tang WH, Wang Z, Levison BS, et al. Intestinal microbial metabolism of phosphatidylcholine and cardiovascular risk. The New England journal of medicine. Apr 25; 2013 368(17):1575-84. PubMed PMID: 23614584. Pubmed Central PMCID: 3701945. This study shows that ingestion of phosphatidylcholine leads to elevated plasma TMAO levels in healthy humans unless their microbiota was depleted first with antibiotics. Next, they show that plasma TMAO levels were associated with increased risk for cardiovascular events in humans with cardiovascular disease risk factors. [PubMed: 23614584]

*31. Koeth RA, Wang Z, Levison BS, et al. Intestinal microbiota metabolism of L-carnitine, a nutrient in red meat, promotes atherosclerosis. Nature medicine. May; 2013 19(5):576-85. PubMed PMID: 23563705. Pubmed Central PMCID: 3650111 This study provides further evidence for 
microbiota metabolism of dietary substrates in the production of the proatherogenic molecule, TMAO. In testing omnivorous and vegan human subjects, the provide evidence for a possible link between microbiota composition, red meat consumption and cardiovascular disease risk.

32. Simren M, Barbara G, Flint HJ, et al. Intestinal microbiota in functional bowel disorders: a Rome foundation report. Gut. Jan; 2013 62(1):159-76. PubMed PMID: 22730468. Pubmed Central PMCID: 3551212. [PubMed: 22730468]

33. Halmos EP, Christophersen CT, Bird AR, et al. Diets that differ in their FODMAP content alter the colonic luminal microenvironment. Gut. Jul 12.2014 PubMed PMID: 25016597.

*34. Halmos EP, Power VA, Shepherd SJ, et al. A diet low in FODMAPs reduces symptoms of irritable bowel syndrome. Gastroenterology. Jan; 2014 146(1):67-75. e5. PubMed PMID: 24076059. In this randomized, controlled, single-blind, cross-over study, a low FOMAP diet improved symptoms in patients with IBS. This is further evidence for the use of low FODMAP diet in the treatment of IBS. [PubMed: 24076059]

35. Tillisch K, Labus J, Kilpatrick L, et al. Consumption of fermented milk product with probiotic modulates brain activity. Gastroenterology. Jun; 2013 144(7):1394-401. 401 e1-4. PubMed PMID: 23474283. Pubmed Central PMCID: 3839572. [PubMed: 23474283]

36. Britton RA, Young VB. Role of the intestinal microbiota in resistance to colonization by Clostridium difficile. Gastroenterology. May; 2014 146(6):1547-53. PubMed PMID: 24503131. Pubmed Central PMCID: 3995857. [PubMed: 24503131]

37. Kassam Z, Lee CH, Yuan Y, et al. Fecal microbiota transplantation for Clostridium difficile infection: systematic review and meta-analysis. The American journal of gastroenterology. Apr; 2013 108(4):500-8. PubMed PMID: 23511459. [PubMed: 23511459]

**38. van Nood E, Vrieze A, Nieuwdorp M, et al. Duodenal infusion of donor feces for recurrent Clostridium difficile. The New England journal of medicine. Jan 31; 2013 368(5):407-15. PubMed PMID: 23323867. In a randomized, controlled trial for the treatment of recurrent Clostridium difficile infection, fecal microbiota transplant plus vancomycin was clearly superior to vancomycin alone. FMT is increasingly recognized as an effective therapy for CDI. [PubMed: 23323867]

*39. Seekatz AM, Aas J, Gessert CE, et al. Recovery of the gut microbiome following fecal microbiota transplantation. mBio. 2014; 5(3):e00893-14. PubMed PMID: 24939885. Pubmed Central PMCID: 4068257. This study describes the resulting microbiota in recipients of FMT for CDI. [PubMed: 24939885]

*40. Petrof EO, Gloor GB, Vanner SJ, et al. Stool substitute transplant therapy for the eradication of Clostridium difficile infection: 'RePOOPulating' the gut. Microbiome. 2013; 1(1):3. PubMed PMID: 24467987. Pubmed Central PMCID: 3869191. As a potential step forward in the use of FMT for recurrent CDI, these authors demonstrate efficacy in the treatment of recurrent CDI for fecal bacterial strains that were cultured and purified before transfer. [PubMed: 24467987]

*41. Theriot CM, Koenigsknecht MJ, Carlson PE Jr. et al. Antibiotic-induced shifts in the mouse gut microbiome and metabolome increase susceptibility to Clostridium difficile infection. Nature communications. 2014; 5:3114. PubMed PMID: 24445449. Pubmed Central PMCID: 3950275. This preclinical study provides new mechanistic information explaining how C. difficile may infect the antibiotic treated host.

42. Kostic AD, Xavier RJ, Gevers D. The microbiome in inflammatory bowel disease: current status and the future ahead. Gastroenterology. May; 2014 146(6):1489-99. PubMed PMID: 24560869. Pubmed Central PMCID: 4034132. [PubMed: 24560869]

**43. Gevers D, Kugathasan S, Denson LA, et al. The treatment-naive microbiome in new-onset Crohn's disease. Cell host \& microbe. Mar 12; 2014 15(3):382-92. PubMed PMID: 24629344. Pubmed Central PMCID: 4059512. Using a large cohort of new-onset, treatment-naive pediatric Crohn's disease patients, this case-control study describes early CD-associated features of the intestinal microbiome. The finding that rectal, but not fecal, microbiota differentiated CD and healthy patients may have diagnostic implications. [PubMed: 24629344]

**44. Haberman Y, Tickle TL, Dexheimer PJ, et al. Pediatric Crohn disease patients exhibit specific ileal transcriptome and microbiome signature. The Journal of clinical investigation. Aug 1; 2014 124(8):3617-33. PubMed PMID: 25003194. Pubmed Central PMCID: 4109533. In a large casecontrol study of new-onset, treatment-naive IBD pediatric patients, the authors identify patterns 
of ileal host gene expression and ileal microbiome that are unique to $\mathrm{CD}$ patients irrespective of the presence of ileal inflammation. These data on early changes in the microbiota and host response may support the hypothesis that dysbiosis contributes to the development of IBD in susceptible individuals. [PubMed: 25003194]

45. Anderson JL, Edney RJ, Whelan K. Systematic review: faecal microbiota transplantation in the management of inflammatory bowel disease. Alimentary pharmacology \& therapeutics. Sep; 2012 36(6):503-16. PubMed PMID: 22827693. [PubMed: 22827693]

46. Kump PK, Grochenig HP, Lackner S, et al. Alteration of intestinal dysbiosis by fecal microbiota transplantation does not induce remission in patients with chronic active ulcerative colitis. Inflammatory bowel diseases. Sep; 2013 19(10):2155-65. PubMed PMID: 23899544. [PubMed: 23899544] 


\section{KEY POINTS}

- The known sequences of the human microbial metagenome likely contain the vast majority of common bacterial species.

- An individual's microbiome is quite stable over time, but there is variability at the extremes of age and among different individuals. Diet and other environmental factors also affect the composition of the microbiome.

- In comparison to healthy controls, alterations in the microbiota are recognized in a growing number of disease states, but, outside of Clostridium difficile infection, the role of these microbiota alterations in the pathogenesis of disease is uncertain.

- A better understanding of the functional interactions between the human host and the microbiome is very likely to lead to new diagnostic, prognostic and therapeutic capabilities. 


\section{Table 1}

Glossary of key terms.

\begin{tabular}{ll}
\hline Term & Definition \\
\hline Richness & the number of distinct members ("species") in the community \\
Diversity & $\begin{array}{l}\text { a measure of the richness and evenness characteristics of a community, often } \\
\text { calculated as a specific "diversity index" }\end{array}$ \\
Dysbiosis & $\begin{array}{l}\text { a term used to refer to a microbiota community associated with a diseased } \\
\text { state that can be differentiated from the microbiota community associated } \\
\text { with a healthy control state }\end{array}$ \\
\hline
\end{tabular}

